\title{
RESIN PERMEABILITY ANALYSIS FOR WOVEN COMPOSITES USING A THREE-SCALE HOMOGENIZATION METHOD
}

\author{
KAZUYA YASUOKA ${ }^{1}$, TETSUYA MATSUDA ${ }^{2}$, \\ TOMOHIRO SAWADA ${ }^{3}$ AND JUNICHI MATSUMOTO ${ }^{4}$ \\ ${ }^{1}$ Department of Engineering Mechanics and Energy, University of Tsukuba \\ 1-1-1 Tennodai, Tsukuba 305-8573, Japan \\ s2020883@s.tsukuba.ac.jp \\ ${ }^{2}$ Department of Engineering Mechanics and Energy, University of Tsukuba \\ 1-1-1 Tennodai, Tsukuba 305-8573, Japan \\ matsuda@kz.tsukuba.ac.jp \\ ${ }^{3}$ Research Center for Computational Design of Advanced Functional Materials, \\ National Institute of Advanced Industrial Science and Technology \\ 1-1-1 Umezono, Tsukuba 305-8568, Japan \\ tomohiro-sawada@aist.go.jp \\ ${ }^{4}$ Research Center for Computational Design of Advanced Functional Materials, \\ National Institute of Advanced Industrial Science and Technology \\ 1-1-1 Umezono, Tsukuba 305-8568, Japan \\ matsumoto-junichi@aist.go.jp
}

Key words: Woven composite, Permeability, Homogenization, Multiscale.

Abstract. In this study, resin permeability of plain-woven composites is analyzed using a three-scale homogenization method. To this end, the three-scale homogenization method is developed to analyze the resin flow through both the structures of fiber bundles and the inside of fiber bundles, and also the mesoscopic and macroscopic resin permeability. To evaluate the validity of this method, resin permeability and characteristic flow velocity of plain-woven glass fiber-reinforced plastic (GFRP) are analyzed. The results show that the present method successfully analyzes the resin permeability of plain-woven composites.

\section{INTRODUCTION}

Woven composites have many positive features, for example, high specific strength, high specific stiffness and good formability compared to single materials such as metals. Therefore, they have been used as primary structural members in many industrial fields such as the aerospace, auto and energy-related industries. In recent years, the resin transfer molding (RTM) has been attracting attention as a manufacturing method for woven composites from the viewpoint of production cost and efficiency. In the RTM, resin materials are impregnated 
through reinforcements consisting of fibers. The RTM method has higher production efficiency compared with the autoclave method, but there is a disadvantage that it may lack stability. Therefore, it is necessary to understand permeation behavior of resin materials through fiber reinforcements in order to further improve the production efficiency and ensure the stability of this molding method. In general, such permeation behavior has been examined experimentally. However, conducting experiments one by one for many kinds of woven composites is not realistic, and it is desirable to predict the permeation behavior analytically.

For such analysis, the mathematical homogenization method $[1,2]$ is one of the most useful methods because the method can analyze both macroscopic and microscopic properties of heterogeneous materials. In fact, the method has been applied to the permeability analysis of woven composites, showing its validity [3]. However, this study did not explicitly consider microscopic structures consisting of fibers and a resin in fiber bundles. On the other hand, an analysis method called the three-scale homogenization method [4] has been developed by our research group. In this method, the whole material is defined as the macroscale, the fiber bundles inside the material is defined as the mesoscale, and the fibers inside the fiber bundle are defined as the microscale. The method allows us to obtain the mechanical properties of composites in more detail by applying the homogenization method twice to the three scales. Thus, the analysis of thermal properties of plain-woven composites using this method was already conducted by the authors [5].

In this study, a resin permeability analysis method for plain-woven composites is developed based on a three-scale homogenization method. To this end, the mathematical homogenization method is applied to resin flow in both bundles and the inside flow velocity distribution glass fiber-reinforced plastic (GFRP) is analyzed

2 THREE-SCALE HOMOGENIZATION METHOD FOR FLUID PROBLEM

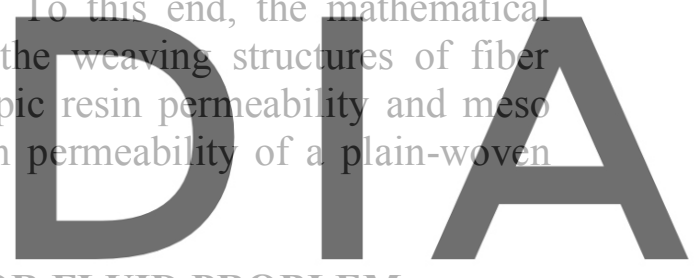
for free at https//www.scipedia.com to download the version without the watermark

For permeability analysis, an analysis model is defined, in which a whole plain-woven composite is regarded as a macro scale structure, fiber bundles (yarn) and resin flow path in the composite as a meso scale structure, and fibers and resin flow path in a fiber bundle as a micro scale structure (Fig.1). A resin permeates the fiber bundle areas, but does not permeates the fiber areas. Both fiber bundles and fibers are treated as rigid bodies. The Cartesian coordinate systems are defined in each scale as illustrated in Fig. 1. Then, we define a meso scale periodic structure as a unit cell $A$, and a micro-scale periodic structure as a unit cell $B$. the density, viscosity and flow velocity of the fluid are defined as $\rho, \mu$ and $u_{i}$, respectively, and the external force per unit mass is defined as $f_{i}$. If the resin is treated as incompressible fluid, the following continuity equation is given.

$$
\frac{\partial u_{i}}{\partial x_{i}}=0
$$

Assuming steady-state flow and a small Reynolds number, the following Stokes equation is given as the equation of motion for incompressible fluid 


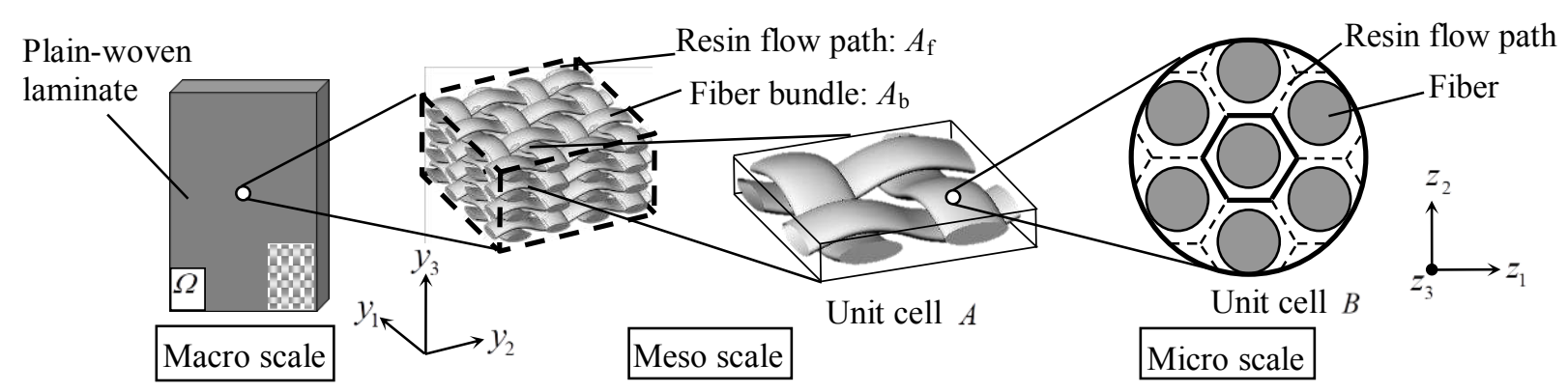

Fig. 1. Plain-woven composite and three-scale structure.

$$
\frac{\partial p}{\partial x_{i}}-\mu \frac{\partial u_{i}}{\partial x_{j}^{2}}=\rho f_{i}
$$

Furthermore, the following basic equations can be given in arbitrary hierarchy

$$
\frac{\partial^{(\varepsilon)} p}{\partial^{(\varepsilon)} x_{i}}-{ }^{(\varepsilon)} \mu \frac{\partial^{(\varepsilon)} u_{i}}{\partial^{(\varepsilon)} x^{2}}=\rho f_{i}
$$
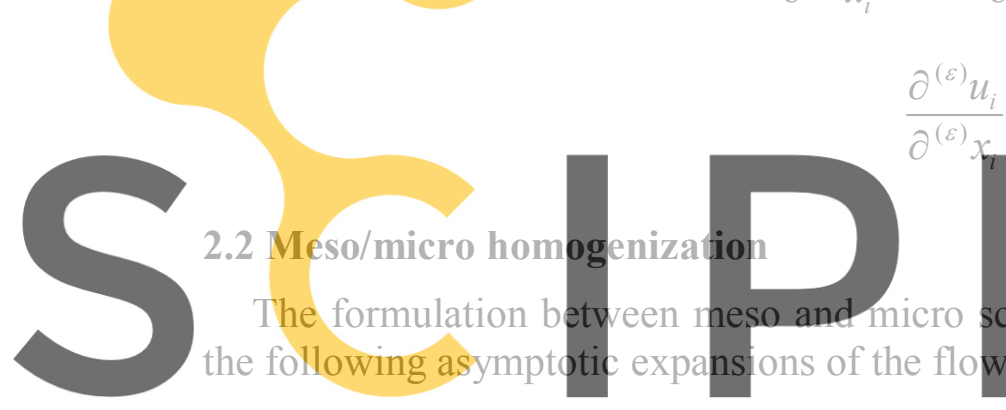

$$
{ }^{(\varepsilon)} u_{i}(y, z)={ }^{(1)} u_{i}(y, z)+\varepsilon^{(2)} u_{i}(y, z)+\varepsilon^{2(3)} u_{i}(y, z)+\cdots,
$$

Register for free at https//www.scipedia.com to download the version without the watermark

where $\varepsilon$ indicates the scale factor between the meso and micro scales. Equations (3)-(6) and the relation $\mu \approx \varepsilon^{2}{ }^{(2)} \mu$ [6] give the governing equations for the micro flow path by considering the identity relations of $\varepsilon$ :

$$
\begin{gathered}
\frac{\partial^{(2)} p}{\partial z_{i}}-{ }^{(2)} \mu \frac{\partial^{2(1)} u_{i}}{\partial z_{j}^{2}}=\rho f_{i}(y)-\frac{\partial^{(1)} p(y)}{\partial y_{i}}, \\
\frac{\partial^{(1)} u_{i}}{\partial z_{i}}=0 .
\end{gathered}
$$

These two equations respectively represent the Stoke equation and the continuity equation for the micro resin flow path. Now, we express ${ }^{(1)} u_{i}$ and ${ }^{(2)} p$ as follow [7]

$$
{ }^{(1)} u_{i}=\frac{1}{{ }^{(2)} \mu}{ }^{(2)} \kappa_{i}^{k}(\boldsymbol{z})\left(\rho f_{k}-\frac{\partial^{(1)} p}{\partial y_{k}}\right),
$$




$$
{ }^{(2)} p={ }^{(2)} \lambda^{k}(\boldsymbol{z})\left(\rho f_{k}-\frac{\partial^{(1)} p}{\partial y_{k}}\right),
$$

where ${ }^{(2)} \kappa_{i}^{k}(\boldsymbol{z})$ and ${ }^{(2)} \lambda^{k}(\boldsymbol{z})$ respectively denote the micro characteristic flow velocity and the micro characteristic pressure which are $Y$-periodic functions. Then, we can derive the following boundary value problem for $B$ by substituting these characteristic functions to Eq. (7), Eq. (8) and the non-slip condition

$$
\begin{gathered}
\frac{\partial^{(2)} \lambda^{k}}{\partial z_{i}}-\frac{\partial^{2(2)} \kappa_{i}^{k}}{\partial z_{j}^{2}}=\delta_{i k}, \\
\frac{\partial^{(2)} \kappa_{i}^{k}}{\partial z_{i}}=0, \\
{ }^{(2)} \kappa_{i}^{k}=0 \text { on } \Gamma_{B},
\end{gathered}
$$

where $\delta_{i k}$ indicates the Kronecker's delta. Furthermore, the following mesoscopic permeability which is homogenized permeability for fiber bundles can be obtained by applying the volume average to the micro characteristic flow velocity

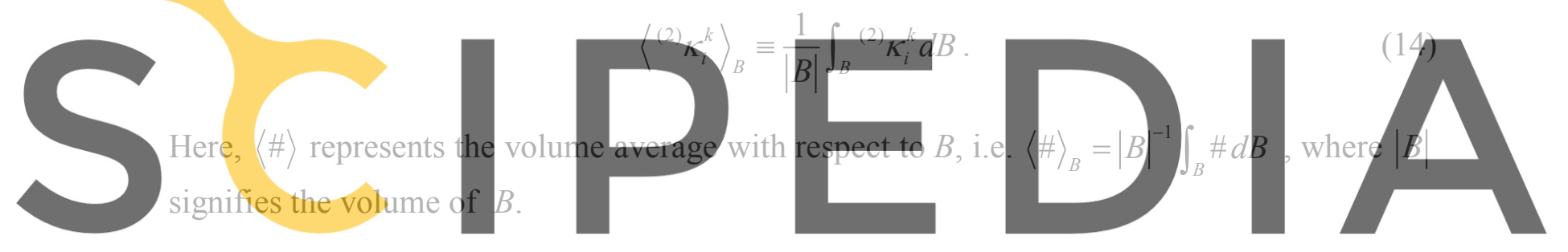

2.3 Macro/meso homogenization

Register for free at https//www.scipedia.com to down.load the version without the watermark The formulation between macro and meso scales is described in this section. In the meso

scale, the resin flows through the meso resin flow path, which is governed by the Stokes equation and the continuity equation, while the resin permeates the fiber bundles. Thus, the following governing equation for the meso fiber bundles is given by substituting the mesoscopic permeability into the relationship between meso and micro flow velocity

$$
\frac{\partial^{(1)} p}{\partial y_{i}}+{ }^{(2)} \mu\left\langle{ }^{(2)} \kappa_{j}^{i}\right\rangle_{B}{ }^{-1}{ }^{(0)} u_{j}=\rho f_{i} .
$$

This equation is called the Brinkman equation. The governing equations for the whole meso scale are obtained by unifying the equations for two regions as follows

$$
\begin{gathered}
\frac{\partial^{(1)} p}{\partial y_{i}}-{ }^{(1)} \mu \frac{\partial^{2(0)} u_{i}}{\partial y_{j}^{2}}+{ }^{(2)} \mu\left\langle{ }^{(2)} \kappa_{j}^{i}\right\rangle_{B}^{-1}{ }^{(0)} u_{j}=\rho f_{i}-\frac{\partial^{(0)} p}{\partial x_{i}} \\
\frac{\partial^{(0)} u_{i}}{\partial y_{i}}=0
\end{gathered}
$$


The boundary value problem for $A$ is obtained as follows by substituting characteristic functions as with the previous meso/micro analysis

$$
\begin{gathered}
\frac{\partial^{(1)} \lambda^{k}}{\partial y_{i}}-\frac{\partial^{2(1)} \kappa_{i}^{k}}{\partial y_{j}^{2}}+\frac{{ }^{(2)} \mu}{{ }^{(1)} \mu}\left\langle{ }^{(1)} \kappa_{j}^{i}\right\rangle_{B}{ }^{-1}{ }^{(1)} \kappa_{j}^{k}=\delta_{i k}, \\
\frac{\partial^{(1)} \kappa_{i}^{k}}{\partial y_{i}}=0
\end{gathered}
$$

where ${ }^{(1)} \kappa_{i}^{k}(\boldsymbol{y})$ and ${ }^{(1)} \lambda^{k}(\boldsymbol{y})$ respectively denote the meso characteristic flow velocity and the meso characteristic pressure which are $Y$-periodic functions. Furthermore, the following macroscopic permeability which is homogenized permeability of the entire composite can be obtained by applying the volume average for $A$ to the meso characteristic flow velocity

$$
\left\langle{ }^{(1)} K_{i}^{k}\right\rangle_{A}=\frac{1}{|A|} \int_{A}^{(1)} K_{i}^{k} d A
$$

\section{MESO/MICRO ANALYSIS}

\subsection{Anallysis conditions}

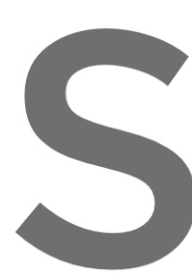

The analysis mode

$72 \%$ was created as sh

elements (18240 ele

periodic boundary cond

condition to the bounda
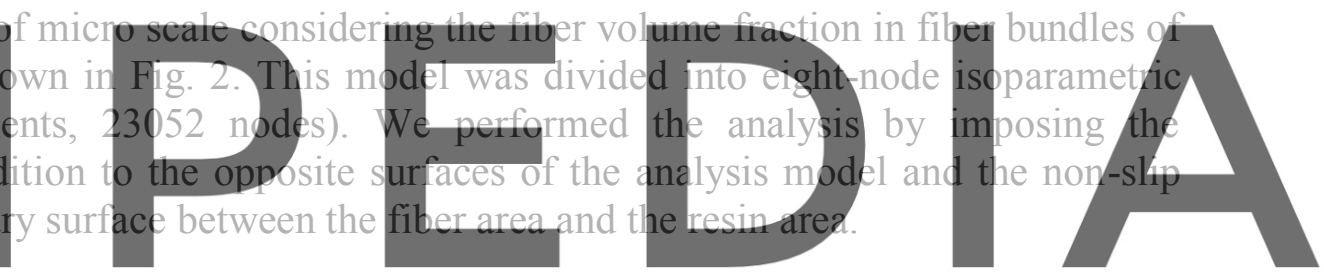

3.2 Results of analysis

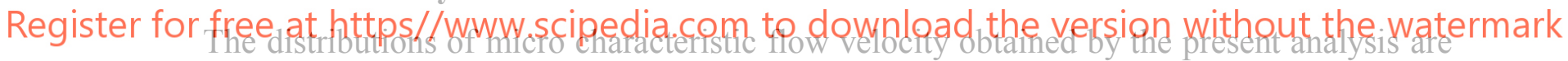

shown in Fig. 3. The characteristic flow velocity distribution is shown by vectors which represent resin flow at the nodes in the finite element model when a unit pressure gradient is given to the macro scale. The meso permeability tensor obtained by averaging these micro characteristic flow velocities for $B$ is as follows

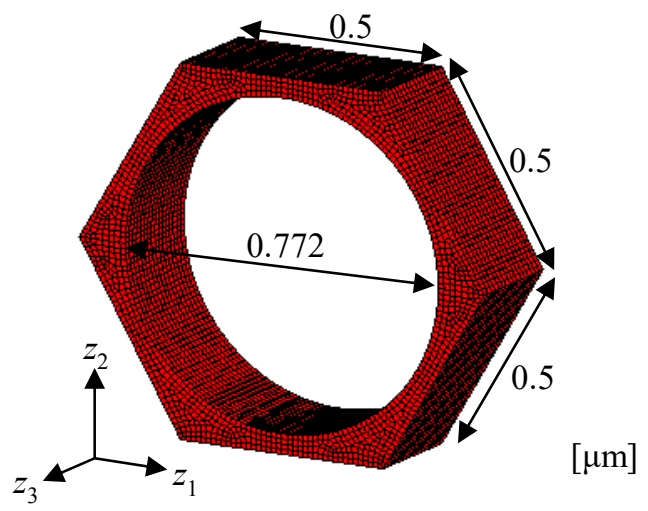

Fig. 2 Unit cell $B$ in micro scale and finite element mesh. 


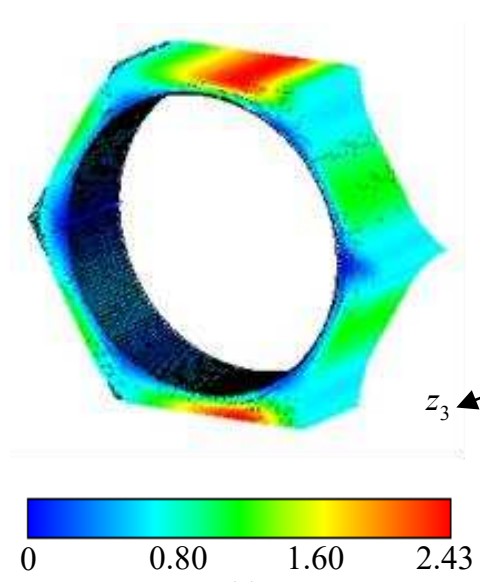

(a)

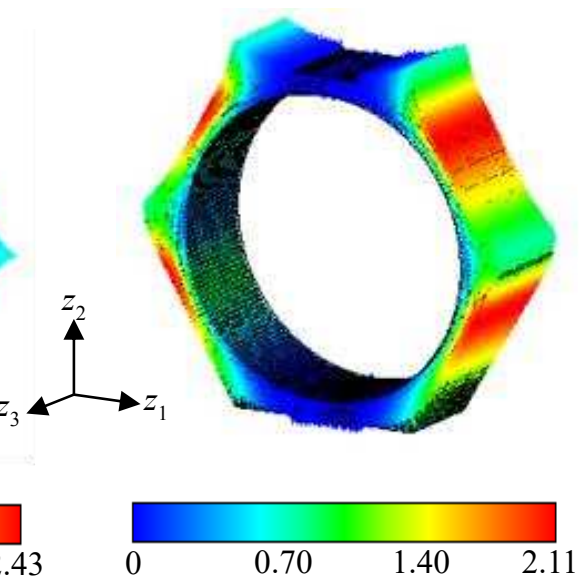

(b)

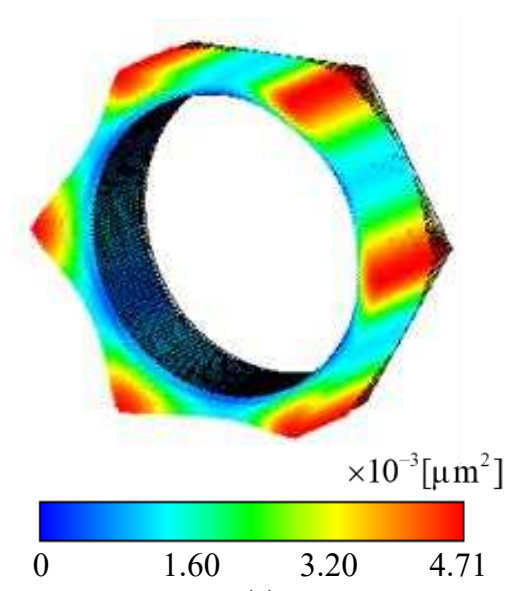

(c)

Fig. 3 Micro characteristic flow velocity distributions in unit cell $B$;

(a) $z_{1}$-direction, (b) $z_{2}$-direction, (c) $z_{3}$-direction.

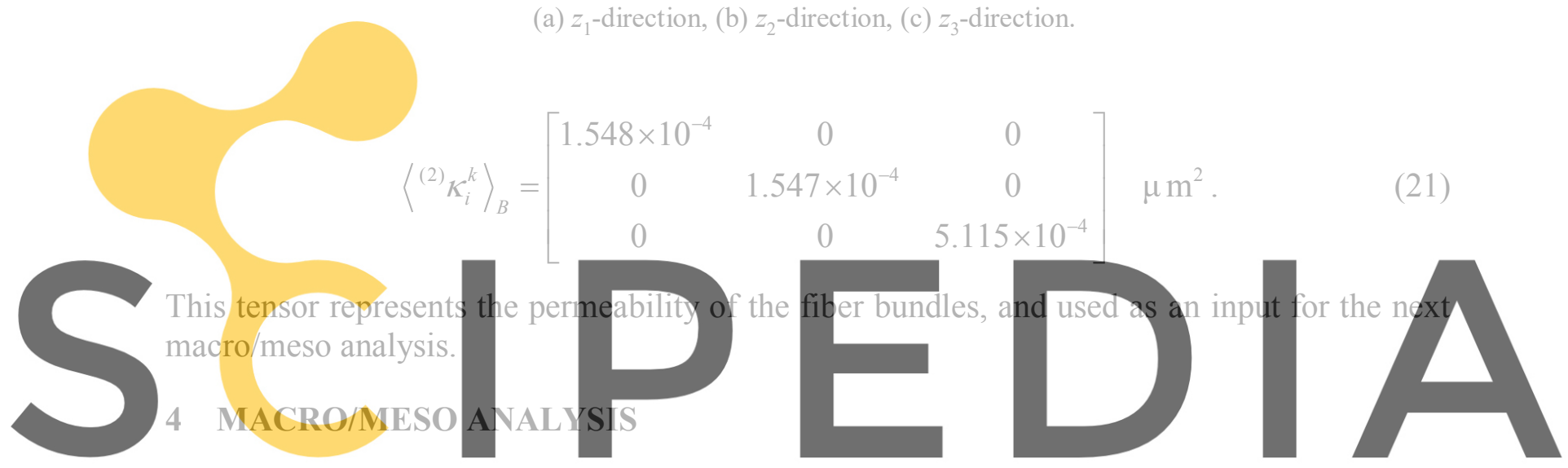

4.1 Analysis conditions

Register for free ath https/dwww. scipedia com ta downdoag the version without the watermark composite of 44\% was created as shown in Fig. 4. This model was divided into eight-node isoparametric elements ( 8192 elements, 9603 nodes). We performed the analysis by imposing the periodic boundary condition to the opposite surfaces of the analysis model. The meso permeability tensor obtained by the previous meso/micro analysis was introduced into this analysis as the permeability of the fiber bundles.
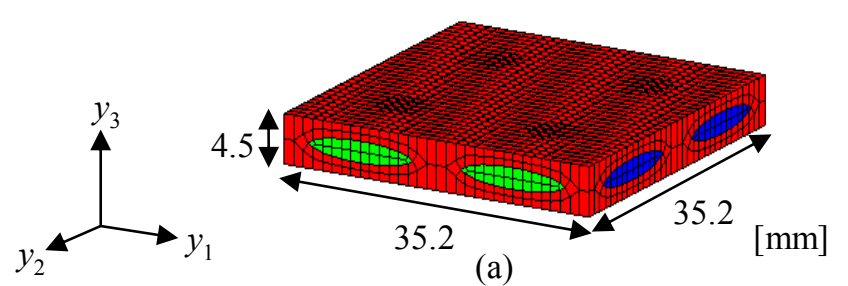

(a)

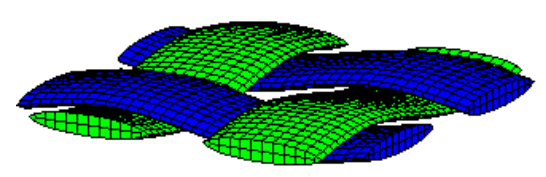

(b)

Fig. 4 Unit cell $A$ in meso scale and finite element mesh;

(a) whole image, (b) fiber bundles. 


\subsection{Results of analysis}

The distributions of meso characteristic flow velocity are shown in Fig. 5. The macro permeability tensor obtained by averaging these meso characteristic flow velocities for $A$ is as follows

$$
\left\langle{ }^{(1)} \kappa_{i}^{k}\right\rangle_{A}=\left[\begin{array}{ccc}
1.721 \times 10^{-1} & 0 & 0 \\
0 & 1.721 \times 10^{-1} & 0 \\
0 & 0 & 1.612 \times 10^{-1}
\end{array}\right] \quad \mathrm{mm}^{2} .
$$

The meso characteristic flow velocity distribution and the macro permeability tensor show isotropy in the $y_{1}$ - and $y_{2}$ - directions due to the symmetry of the structure in these directions. In the $y_{3}$-direction, on the other hand, the concentration of the characteristic flow velocity through the meso pores, and the greater value compared to the previous experimental research for various woven structures $[8,9]$ can be confirmed. One of the reasons is that the actual woven composites have laminate misalignment mainly caused by the pressure during molding.

\section{CONCLUSIONS}

The three-scale homogenization method was developed to be applied to the resin flow problem in woven composites. The present method enabled us to analyze the characteristic flow velocity distribution and the resin permeability from multiscale

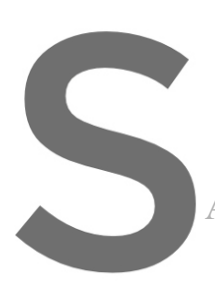
perspective. The characteris woven GFRP

ACINOWLEDGMENTS

This presentation is
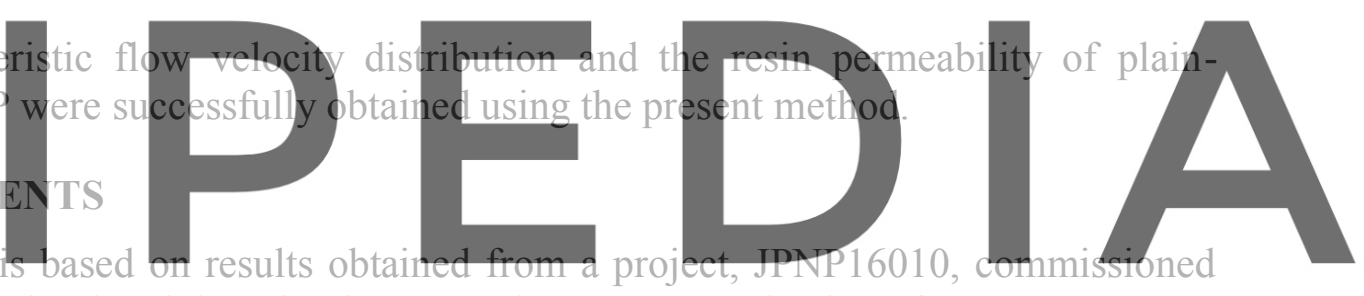

by the New Energy and Industrial Technology Development Organization of Japan (NEDO).

Register for free at https//www.scipedia.com to download the version without the watermark REFERENCES

[1] Bensoussan, A., Lions, J.L. and Papanicolau, G. Asymptotic analysis for periodic structures. North Holland, (1978).

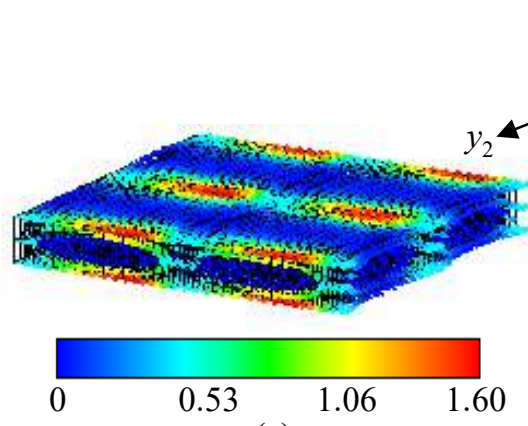

(a)

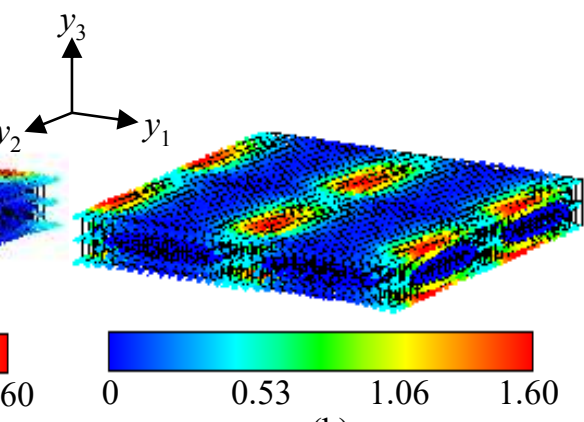

(b)

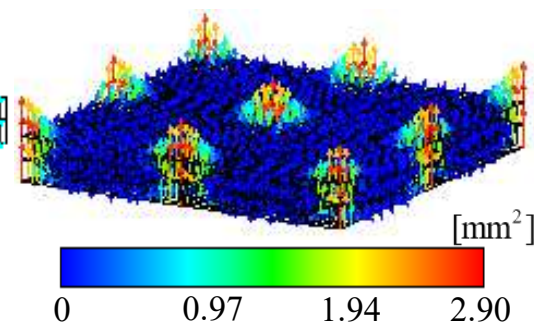

(c)

Fig. 5 Meso characteristic flow velocity distributions in unit cell $A$;

(a) $y_{1}$-direction, (b) $y_{2}$-direction, (c) $y_{3}$-direction. 
[2] Sanchez-Palencia, E. Non-homogeneous Media and Vibration Theory, Lecture Notes in Physics, Vol. 127. Springer-Verlag, (1980).

[3] Takano, N., Zako, M., Okazaki, T. and Terada, K. Microstructure-based evaluation of the influence of woven architecture on permeability by asymptotic homogenization theory. Compos. Sci. Technol. (2002) 62:1347-1356.

[4] Oide, K. and Matsuda, T. Macro/meso/micro elastic-viscoplastic analysis of plain-woven laminates using homogenization theory. Key Eng. Mater. (2015) 626:365-371.

[5] Sato, Y. and Matsuda, T. Evaluation of micro/meso/macro thermal properties of plainwoven laminates. Key Eng. Mater. (2017) 725:439-444.

[6] Hornung, U. Homogenization and Porous media. Splinger-Verlag, (1997).

[7] Sawada, T., Nakasumi, S., Tezuka, A. and Yoshizawa, I. Extended finite element discretization technique of the solid-fluid mixture homogenization method with applications to BCC and FCC microstructures. Interact. multiscale Mech. (2009) 2:45-68.

[8] Parnas, S.R., Flynn, M.K. and Dal-favero, E.M. A permeability database for composites manufacturing. Polym. Compos. (1997) 18:623-633.

[9] Naik, K.N., Sirisha, M. and Inani, A. Permeability characterization of polymer matrix composites by RTM/VARTM. Prog. Aerosp. Sci. (2014) 65:22-40.
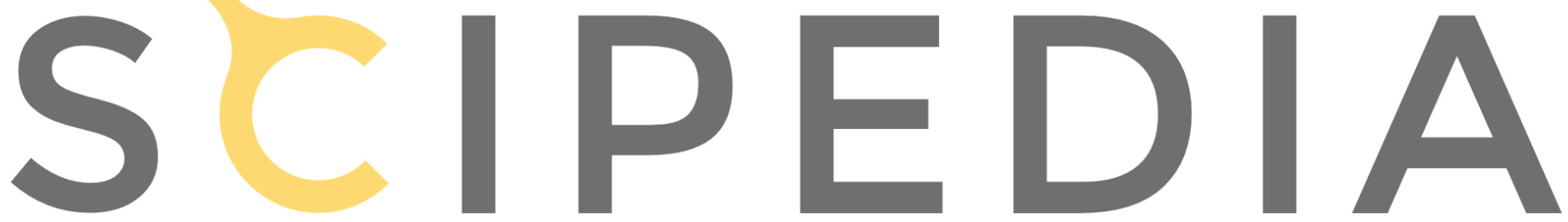

Register for free at https//www.scipedia.com to download the version without the watermark 